\title{
Identification of PDCDI and PDCDILG2 as Prognostic Biomarkers and Associated with Immune Infiltration in Hepatocellular Carcinoma
}

\author{
Wang $\mathrm{Li}^{1,2, *}$ \\ Mei Mei ${ }^{1,3-5, *}$ \\ Tian Liul,* \\ ShuWen Zhang' \\ ZeYu Wang' \\ YuHong Suo' \\ Shuai Wang' \\ Yang Liu' \\ NingNing Zhang' \\ Wei Lu' \\ 'Department of Hepatobiliary Oncology, \\ Liver Cancer Center, Tianjin Medical \\ University Cancer Institute and Hospital, \\ National Clinical Research Center for \\ Cancer, Key Laboratory of Cancer \\ Prevention and Therapy, Tianjin Medical \\ University, Tianjin, 300060, People's Republic \\ of China; ${ }^{2} \mathrm{NHC}$ Key Laboratory of \\ Hormones and Development, Tianjin Key \\ Laboratory of Metabolic Diseases, Chu \\ Hsien-I Memorial Hospital \& Tianjin Institute \\ of Endocrinology, Tianjin Medical University, \\ Tianjin, 300I34, People's Republic of China; \\ ${ }^{3}$ Graduate School of Tianjin Medical \\ University, Tianjin, 300070, People's Republic \\ of China; ${ }^{4}$ Tianjin First Central Hospital, \\ Tianjin, 300192, People's Republic of China; \\ ${ }^{5}$ Department of Gastroenterology, Tianjin \\ Haihe Hospital, Tianjin, 300350, People's \\ Republic of China \\ *These authors contributed equally to this \\ work
}

Correspondence: Wei Lu; NingNing Zhang Department of Hepatobiliary Oncology, Liver Cancer Center, Tianjin Medical University Cancer Institute and Hospital, National Clinical Research Center for Cancer, Key Laboratory of Cancer

Prevention and Therapy, Tianjin Medical University, Tianjin, 300060, People's Republic of China

Email mail4luwei@163.com;

mail4ningning@163.com
Background: Hepatocellular carcinoma has been identified to be among the most prevalent malignancies in the world and has an unfavorable prognosis. Immune checkpoints perform an essential function in many biological processes and are associated with the survival of cancer patients. The function of immune checkpoints remains unknown.

Methods: We used bioinformatic methods to examine the prognostic value of immune checkpoints and the corresponding link to immune infiltration in HCC. qRT-PCR was used to validate the expression of immune checkpoints and their prognostic significance in HCC. Results: The level of mRNA of SIGLEC15, PDCD1LG2, LAG3, PDCD1, CTLA4 as well as PDCD1LG2 was increased in HCC tissues as opposed to liver tissues. Immune checkpoints were shown to participate in the activation of the apoptotic pathway in HCC patients. The elevated expression of PDCD1 and PDCD1LG2 were shown to have a favorable recurrence-free survival (RFS), progression-free survival (PFS), disease-specific survival (DSS), and overall survival (OS). PDCD1, PDCD1LG2, and pT stage were independent variables that affect the HCC patients' prognoses as revealed by the multivariate and univariate analyses. A prediction nomogram indicated that the calibration plots for OS rates over three and five years had a stronger predictive performance in the TCGA HCC cohort in contrast with an ideal model. Positive correlations were observed between the PDCD1 and PDCD1LG2 expression and immune biomarkers, immune cells, chemokine receptors, as well as chemokines.

Conclusion: The present research performed a thorough examination of the prognostic significance of immune checkpoints in HCC and its correlation with immune infiltration, which suggested that PDCD1 and PDCD1LG2 were prognostic biomarkers in HCC and related to the immune infiltration.

Keywords: HCC, PDCD1, PDCD1LG2, immune checkpoints, prognosis biomarker

\section{Introduction}

Owing to its high prevalence and poor prognosis, hepatocellular carcinoma (HCC) has caused the second-highest cancer fatality rate globally. ${ }^{1} \mathrm{HCC}$ is associated with a number of known risk factors, such as metabolic liver disease (especially nonalcoholic fatty liver disease), alcoholism, chronic hepatitis $\mathrm{B}$ and $\mathrm{C}$ infection, and exposure to food toxins that include aristolochic and aflatoxins acid. Treatment options for HCC include thermal ablation (heat treatment), surgical resection, radiotherapy (radiation therapy), and liver transplantation (for individuals with early-stage disease). ${ }^{2}$ Patients with middle-stage HCC are treated with catheter-based locoregional therapy. In 
addition, for patients with advanced-stage HCC, it has been established that kinase and immune checkpoint inhibitors are viable therapy alternatives. ${ }^{3}$

Immune checkpoint molecules that include both stimulatory and inhibitory immune checkpoint molecules are considered ligand-receptor combinations that block or stimulate immune responses. A growing body of research evidence has illustrated that immune checkpoint molecules, regardless of stimulatory or inhibitory ones, are significantly expressed in high proportion in distinct types of tumors. Despite the fact that the primary role of immune checkpoint molecules associated with tumor cells is considered to be the facilitation of immune escape, it was recently demonstrated that these molecules perform essential functions in the maintenance of a variety of mechanisms, such as drug resistance, improved energy metabolisms, selfrenewal, metastasis, anti-apoptosis, angiogenesis, or even the epithelial-mesenchymal transition. ${ }^{4}$ Moreover, another study suggested that loss of histone macroH2A1 could activate CD4 + CD25 + FoxP3 + regulatory $\mathrm{T}$ cells and improve paracrine-mediated chemoresistance in $\mathrm{HCC}^{5}$ Recently, inhibitory checkpoints, such as cytotoxic T lymphocyte-associated antigen 4 (CTLA-4), programmed cell death protein-1 (PD-1), and also programmed cell death ligand 1 (PD-L1), have been discovered to attenuate antitumor immune responses in solid tumors. In addition, B7-1 and B7-2 are the co-stimulatory immune checkpoints in HCC that have attracted much attention. ${ }^{6}$ However, there has been no thorough and systematic research on the prognostic significance of immune checkpoint inhibitors as well as their corresponding correlation with immune infiltration in $\mathrm{HCC}^{5}$

Immune checkpoints and their relative association with immune infiltration in human hepatocellular cancer (HCC) are investigated in the present using bioinformatics methods. This may provide new ideas for screening therapeutic targets for HCC.

\section{Materials and Methods}

\section{Dataset and Preprocessing}

The immune checkpoint gene expression profile was obtained from The Cancer Genome Atlas (TCGA) database (https://cancergenome.nih.gov/). To perform additional investigations, the TCGA HCC dataset $(n=374)$ and related clinical features of HCC patients were acquired. The levels of immune checkpoints mRNA were analyzed and visualized by $\mathrm{R}$ (version 4.0.3) with packages ggplot2 and pheatmap.

\section{Drug Sensitivity, Genetic Mutation, and Cancer-Related Pathway Analysis}

Using the gene mutation data collected from the TCGA HCC dataset, we visualized the genetic mutation of eight immune checkpoints utilizing the "maftools" package in R. Using the Genomics of Drug Sensitivity in Cancer (GDSC), we obtained data on a sum of 265 small moles or drugs. Using Pearson correlation analysis, we then determine the correlation coefficient between immune checkpoints and these small compounds or drugs. The HCC cohort was classified into two groups with a medium level of immune checkpoints expression. in the case when PAS (immune checkpoint group High) > PAS (immune checkpoint group Low), we hypothesized that immune checkpoints may either activate or block a pathway. According to Ye et al, the approach of cancerrelated pathway analysis was conducted. ${ }^{7}$

\section{Prognosis Analysis and Predictive Nomogram}

Kaplan-Meier survival analysis was utilized to delve into the prognostic significance of immune checkpoints in HCC. The p-values as well as a hazard ratio (HR) having 95\% confidence intervals (CI)were analyzed using the Log rank test. The variables influencing the prognosis of HCC patients were found using univariate and multivariate cox regression analyses, and a prediction nomogram was developed on the basis of these factors. The "forestplot" $\mathrm{R}$ tool was utilized to depict the HR, P-value, and 95\% CI of these components.

\section{The Human Protein Altas}

The Human Protein Altas (https://www.proteinatlas.org/) is a web-based database for performing extensive protein analyses in human malignancy. ${ }^{8}$ The tissue atlas and pathology atlas modules were used in the present research to examine the protein levels of immune checkpoints in liver and HCC tissues.

\section{qRT-PCR}

Tianjin Medical University Cancer Institute and Hospital's Ethics Committee approved the present research. A total of $40 \mathrm{HCC}$ samples and normal liver tissues was acquired from patients who had provided informed consent. Three qualified pathologists assessed the tumor grade and histological 


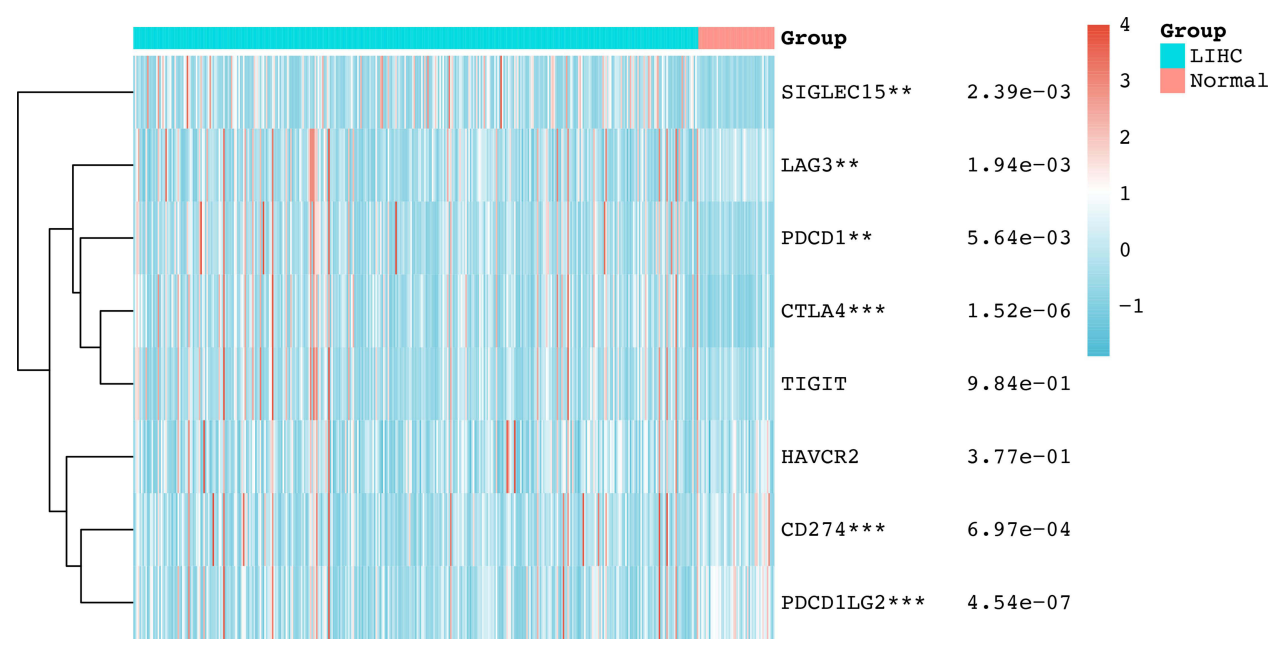

Figure I The mRNA level of immune checkpoints in KIRC. The graph shows the mRNA level of immune checkpoints in HCC tissues compared with normal tissues. **P < 0.01 , *** $* 0.001$.

diagnosis using the American Joint Committee on Cancer (AJCC) staging method released in 2010. We used the TRIzol reagent (from Invitrogen) for the purpose of extracting whole-cell RNA from clinical tissues. cDNA was generated utilizing PrimeScript RT-polymerase in accordance with the manufacturer's specifications (Vazyme). Subsequently, SYBR-Green Premix (Qiagen $\mathrm{GmbH}$ ) was utilized to conduct RT-qPCR with the internal control used in the present study selected as Glyceraldehyde-3-phosphate dehydrogenase (GAPDH). The $2^{-\Delta \Delta C t}$ technique was used to determine the target genes expression.

\section{Infiltration of the Immune Checkpoints}

TIMER (https://cistrome.shinyapps.io/timer/), a web-based platform that allows for in-depth analyses of tumorinfiltrating immune cells, ${ }^{9}$ was employed to evaluate the immune infiltration of immune checkpoints in HCC. The association between immune checkpoints and immune cell infiltration, as well as the expression of immune biomarkers, chemokines, and chemokines receptors, was examined by means of Pearson analysis, with a p-value of 0.05 serving as the cut-off value.

\section{Functional Enrichment Analysis}

HCC immune checkpoints were analyzed using LinkedOmics (http://www.linkedomics.org/adminphp), a web server that allows for extensive analyses. ${ }^{9}$ With a false discovery rate (FDR) of 0.05 , these immune checkpoints were submitted to the gene isolation service LinkedOmics for subsequent analysis. Subsequently, Gene Set Enrichment Analysis (GSEA) was utilized for the purpose of identifying immune checkpoint-related activities, such as analyses on gene ontology (GO) and Kyoto encyclopedia of genes and genomes (KEGG) pathways. In the present research, the minimum number of genes (Size) was set at three whereas the maximum number of simulations was set at five hundred.

\section{Results}

\section{The Expression of Immune Checkpoints in $\mathrm{HCC}$}

Initial detection of immune checkpoint expression in HCC enabled us to better understand the function of immune checkpoints in this disease. According to the TCGA HCC cohort data, immune checkpoint mRNA expression demonstrated that the expression of SIGLEC15( $\mathrm{p}=2.39 \mathrm{e}-5)$, PDCD1LG2 $(\mathrm{p}=2.39 \mathrm{e}-13)$, LAG3 $(\mathrm{p}=1.94 \mathrm{e}-3)$, PDCD1 $(\mathrm{p}=5.64 \mathrm{e}-3)$, CTLA4 $(p=1.52 \mathrm{e}-6)$ and PDCD1LG2 $(\mathrm{p}=4.54 \mathrm{e}-7)$ was elevated in HCC tissues in contrast with liver tissues (Figure 1). Therefore, extensive alteration of immune checkpoints in $\mathrm{HCC}$.

\section{Analyses of Immune Checkpoints in HCC Using Cancer Hallmarks}

Furthermore, we used cancer signature analysis to probe into the function of immune checkpoints in HCC. The genetic alteration immune checkpoints in TCGA HCC patients were comprised of missense mutations, and Del mutations (Figure 2A). SNPs were the most prevalent variation type whereas $\mathrm{C}>\mathrm{A}$ was the most prevalent $\mathrm{SNV}$ type, according to the data (Figure 2A). Across all the immune checkpoints, LAG3 was the most common gene 

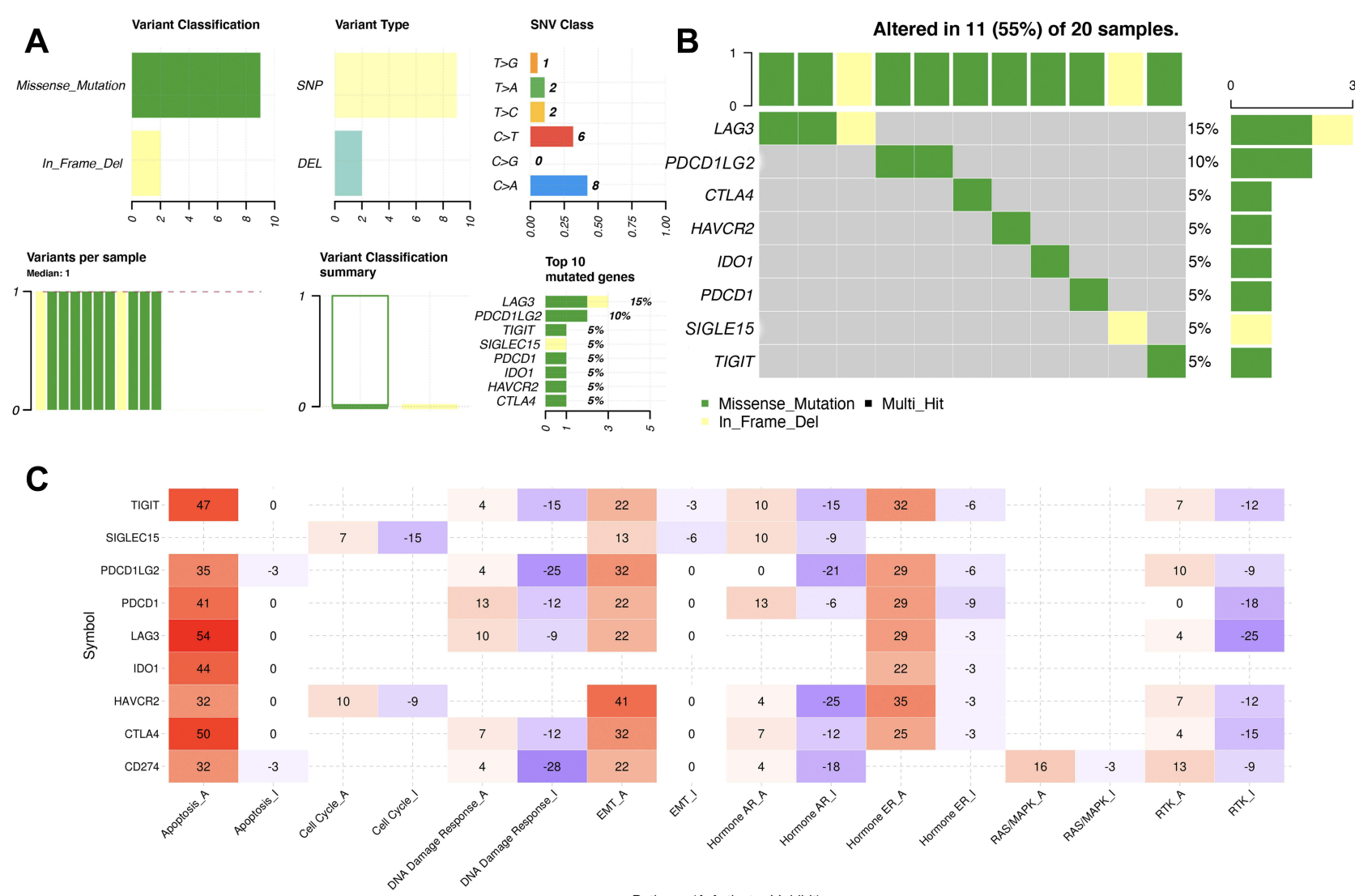

Pathway (A:Activate; I:Inhibit)

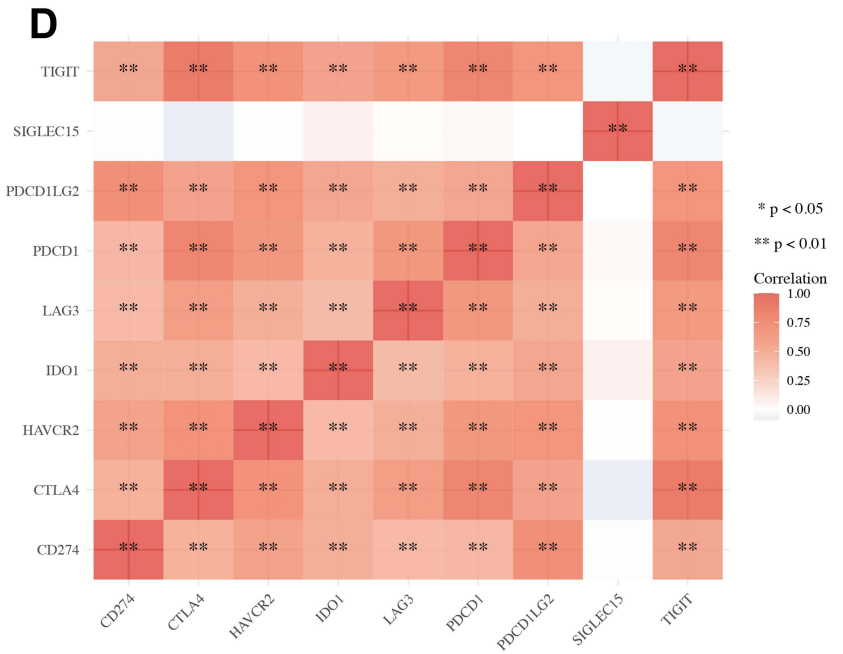

Percent $\begin{array}{rrrr}-25 & 0 & 25 & 50\end{array}$

E

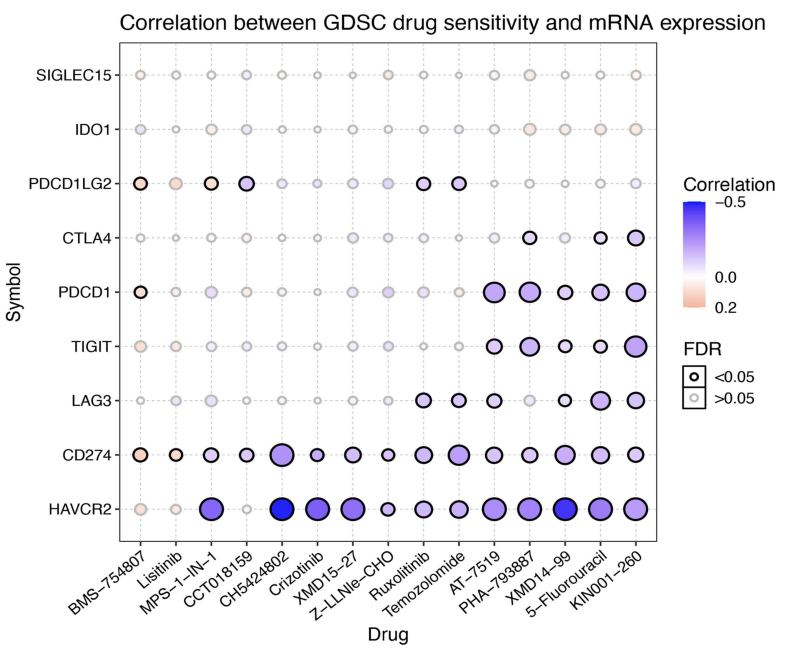

Figure 2 Cancer hallmark of immune checkpoints in HCC. (A and B) Oncoplot displaying genetic mutation landscape of immune checkpoints in TCGA HCC cohort. (C) The activation or inhibition of immune checkpoints in famous cancer-related pathways. (D) Correlation analysis between each member of immune checkpoints. (E) The correlation between immune checkpoints and drug or small molecules. The positive correlation means that the gene high expression is resistant to the drug, vise verse. $* \mathrm{P}<$ $0.05, * * \mathrm{P}<0.01$.

and about $15 \%$ of TCGA HCC cases experience LAG3 genetic mutation (Figure 2B). The hallmark pathways of cancer, such as RTK, Cell Cycle, Hormone ER, PI3K/ AKT, Hormone AR, RAS/MAPK, DNA Damage Response, EMT, TSC/mTOR, as well as apoptosis pathways, perform a significant function in the onset and progression of tumors. According to a correlation study between immune checkpoints and cancer hallmark pathways, immune checkpoints in HCC participated in the activation of the apoptotic, Hormone ER, EMT, as well 
as the DNA damage inhibition response pathways (Figure 2C). Furthermore, co-expression analysis indicated a strong association among every member of immune checkpoints except for SIGLEC15 (Figure 2D). In general, one of the most important steps in the development of therapeutic drugs is to examine the relationship between the genes and the drug targets that already exist. A drug sensitivity analysis performed in the present research demonstrated that some immune checkpoints were susceptible to certain small molecules or medicines from the GDSC (negative association) (Figure 2E).

\section{Prognostic Significance of Immune Checkpoints in HCC}

Previous study had revealed that some immune checkpoints might act as prognostic biomarker in many malignances. In our study, prognosis analysis indicated that PDCD1 and PDCD1LG2 were related to the progression free survival (PFS), disease-specific survival (DSS), overall survival (OS), and recurrence-free survival (RFS) of HCC patients (Tables 1 and 2 and Figure 3). Specifically, HCC patients that exhibited elevated PDCD1 expression showed a better OS $(\mathrm{p}=0.043$, HR $(95 \% \mathrm{CI})=0.68(0.46$ $0.99))$, better RFS $(\mathrm{p}=0.0031$, HR $(95 \% \mathrm{CI})=0.61(0.44$ $0.85))$, better PFS $(\mathrm{p}=0.0027, \mathrm{HR}(95 \% \mathrm{CI})=0.63(0.47-$ $0.86)$ ), and better DSS $(\mathrm{p}=0.013$, HR $(95 \% \mathrm{CI})=0.56$ (0.35-0.89)) (Tables 1 and 2 and Figure 3A). Moreover, HCC patients with high PDCD1LG2 expression showed a better OS $(p=0.00022$, HR $(95 \%$ CI $)=0.5(0.34-0.73))$, better RFS $(\mathrm{p}=0.51 \mathrm{e}-5$, HR $(95 \% \mathrm{CI})=0.51(0.36-0.71))$, better PFS $(\mathrm{p}=1 \mathrm{e}-5$, HR $(95 \% \mathrm{CI})=0.52(0.38-0.7))$, and better DSS $(\mathrm{p}=0.0025$, HR $(95 \% \mathrm{CI})=0.48(0.29-0.78))$ (Tables 1 and 2 and Figure 3B). Thus, PDCD1 and
PDCD1LG2 may serve as prognostic biomarkers in the treatment of HCC.

\section{The Development of a Prediction Nomogram Predicated on Clinicopathologic Characteristics and Immune Checkpoints}

Univariate and multivariable analyses demonstrated that PDCD1, PDCD1LG2, and the stage pT were all independent variables influencing the prognosis of HCC patients (Figure 4A and B). Based on clinicopathologic characteristics (pT stage) as well as potential prognostic biomarkers (PDCD1 and PDCD1LG2), we then used the cox regression technique to generate a predictive nomogram for OS over one, three, and five years in the discovery group. Furthermore, the calibration plots for OS over three and five years were anticipated more accurately in comparison with an ideal model in the TCGA HCC cohort (Figure 4C and D).

\section{Association Between Immune Checkpoints and Clinical Characteristics in $\mathrm{HCC}$}

To thoroughly examine the role of PDCD1 and PDCD1LG2 in HCC, we conducted a correlation analysis between PDCD1/PDCD1LG2 and the clinical characteristics of HCC. It was discovered that HCC patients with a strong tumor grade expressed more PDCD1 as opposed to individuals with a lower tumor grade (Supplementary Figure 1A, $p=0.0017)$. As shown in Supplementary Figure 1B, patients with HCC who had distant tumor metastases exhibited a significantly elevated level of

Table I Prognosis Value of Immune Checkpoints in STAD for Overall Survival and Recurrence-Free Survival

\begin{tabular}{|c|c|c|c|c|}
\hline \multirow[t]{2}{*}{ Genes } & \multicolumn{2}{|c|}{ Overall Survival } & \multicolumn{2}{|c|}{ Recurrence-Free Survival } \\
\hline & p-value & HR $(95 \% \mathrm{CI})$ & p-value & HR (95\% CI) \\
\hline SIGLECI 5 & 0.02 & $0.65(0.45-0.94)$ & 0.11 & $0.79(0.59-1.05)$ \\
\hline IDOI & 0.21 & $0.8(0.57-1.13)$ & 0.054 & $0.7(0.49-1.01)$ \\
\hline CD274 & 0.095 & $0.71(0.47-1.06)$ & 0.23 & $0.81(0.56-1.15)$ \\
\hline HAVCR2 & 0.23 & $1.28(0.86-1.92)$ & 0.02 & $0.66(0.47-0.94)$ \\
\hline PDCDI & 0.043 & $0.68(0.46-0.99)$ & 0.0031 & $0.61(0.44-0.85)$ \\
\hline CTLA4 & 0.018 & $0.65(0.46-0.93)$ & 0.018 & $0.65(0.46-0.93)$ \\
\hline LAG3 & 0.022 & $0.6(0.39-0.93)$ & 0.016 & $0.67(0.48-0.93)$ \\
\hline PDCDILG2 & 0.00022 & $0.5(0.34-0.73)$ & 5.le-05 & $0.5 I(0.36-0.7 I)$ \\
\hline TIGIT & 0.067 & $0.71(0.5-1.03)$ & 0.0012 & $0.58(0.42-0.81)$ \\
\hline
\end{tabular}


Table 2 Prognosis Value of Immune Checkpoints in STAD for Progression Free Survivial and Disease-Specific Survival

\begin{tabular}{|l|l|l|l|l|}
\hline \multirow{2}{*}{ Genes } & \multicolumn{2}{l|}{ Progression Free Survival } & \multicolumn{2}{l|}{ Disease-Specific Survival } \\
\cline { 2 - 5 } & p-value & HR $\mathbf{9 5 \%} \mathbf{~ C I})$ & p-value & HR (95\% CI) \\
\hline SIGLECI5 & 0.029 & $0.69(0.49-0.96)$ & 0.01 I & $0.54(0.33-0.87)$ \\
IDOI & 0.019 & $0.68(0.49-0.94)$ & 0.21 & $1.35(0.85-2.13)$ \\
CD274 & 0.27 & $0.84(0.62-1.14)$ & 0.095 & $0.64(0.38-1.09)$ \\
HAVCR2 & 0.02 & $0.7(0.51-0.95)$ & 0.34 & $0.79(0.48-1.29)$ \\
PDCDI & 0.0027 & $0.63(0.47-0.86)$ & 0.013 & $0.56(0.35-0.89)$ \\
CTLA4 & 0.04 & $0.71(0.52-0.99)$ & 0.31 & $0.78(0.49-1.25)$ \\
LAG3 & 0.0073 & $0.66(0.48-0.9)$ & 0.16 & $0.68(0.4-1.17)$ \\
PDCDILG2 & le-05 & $0.52(0.38-0.7)$ & 0.0025 & $0.48(0.29-0.78)$ \\
TIGIT & 0.0015 & $0.6(0.44-0.83)$ & 0.044 & $0.62(0.39-0.99)$ \\
\hline
\end{tabular}

PDCD1LG2 expression than those who did not develop distant tumor metastases $(\mathrm{p}=0.0036)$.

\section{Validation of PDCDI and PDCDILG2 Expression and Overall Survival in HCC} The expression of PDCD1 and PDCD1LG2 proteins in HCC and normal liver tissues were then investigated. As expected, PDCD1 was high staining in HCC tissues while it was low staining in normal liver tissues (Figure 5A). PDCD1LG2 was low staining in HCC tissues while it was high staining in normal liver tissues (Figure 5B). As shown in Figure 6A and
$\mathrm{B}$, the result of qRT-PCR revealed that the mRNA level of PDCD1 ( $<<0.001)$ was upmodulated as the levels of mRNA of PDCD1LG2 was downmodulated in HCC versus liver tissues $(\mathrm{p}<0.001)$. Further prognosis indicated that HCC patients with elevated levels of PDCD1 (Figure 6C, $\mathrm{p}=0.0142$ ) and PDCD1LG2 (Figure 6D, $\mathrm{p}=0.0252$ ) expression showed improved overall survival. Moreover, the multivariate and univariate analyses demonstrated PDCD1, PDCD1LG2 as well as clinical stage as prognosis factors influencing the $\mathrm{OS}$ of $\mathrm{HCC}$ patients (Figure 6E and F). These data were consistent with findings in other studies.
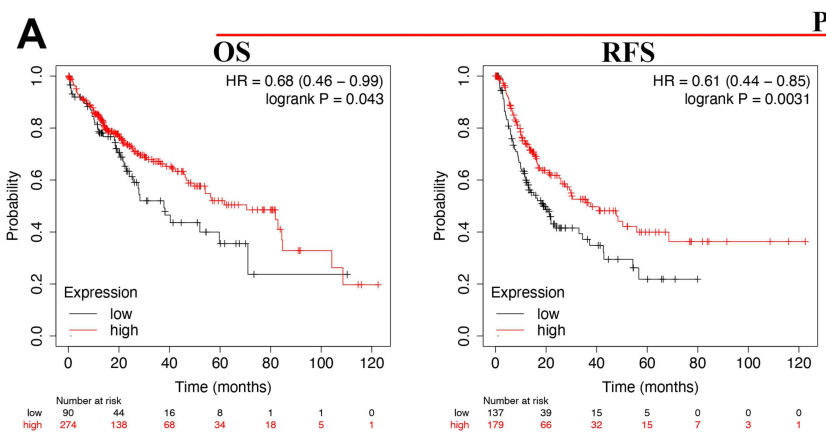

PDCD1
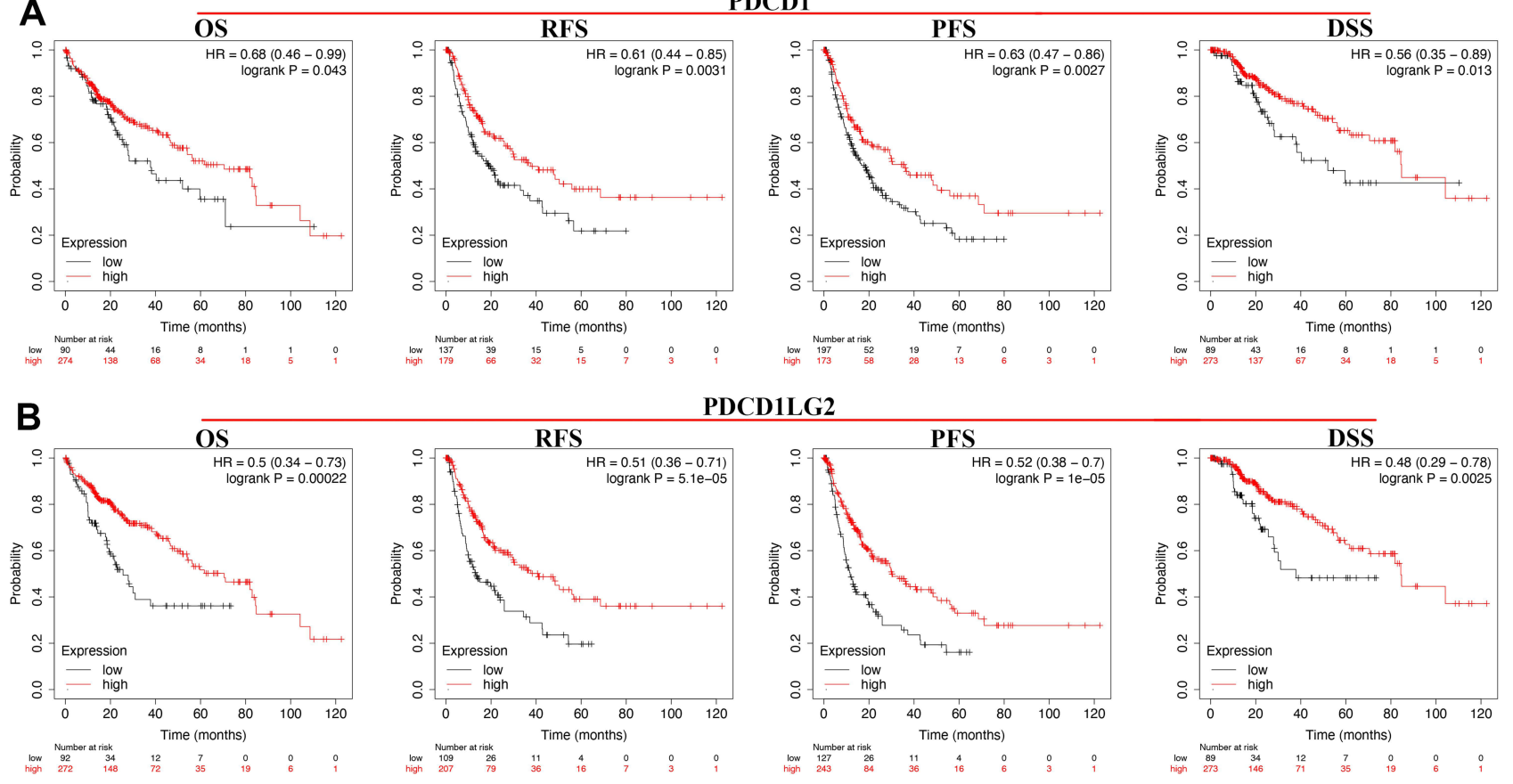

PDCD1LG2

Figure 3 The prognosis analysis of immune checkpoints in HCC. (A) Survival curve of HCC patients with high and low PDCDI expression in overall survival, recurrencefree survival, progression free survival, and disease-specific survival analysis. (B) Survival curve of HCC patients with high and low PDCDILG2 expression in overall survival, recurrence-free survival, progression free survival, and disease-specific survival analysis.

Abbreviations: OS, overall survival; RFS, recurrence-free survival; PFS, progression free survival; DSS, disease-specific survival. 
A

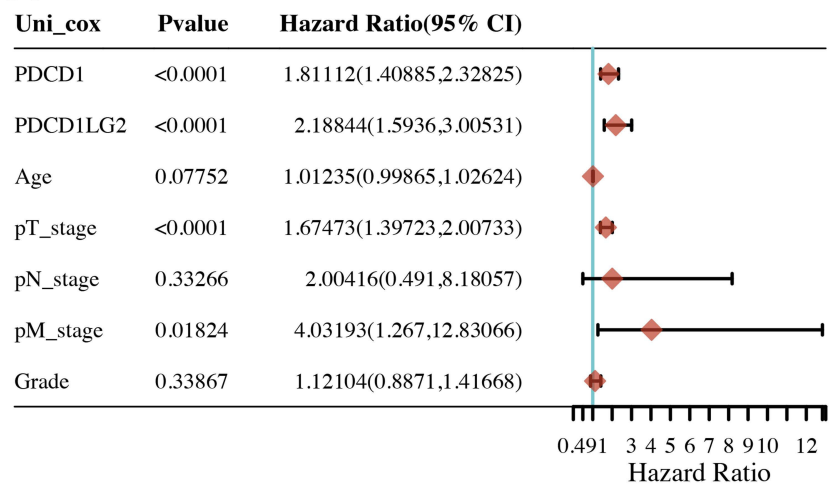

B

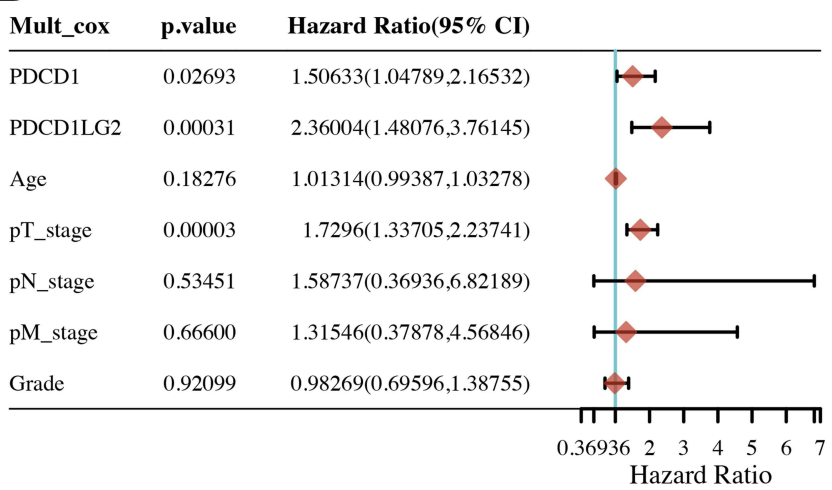

D

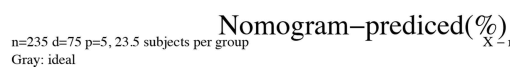

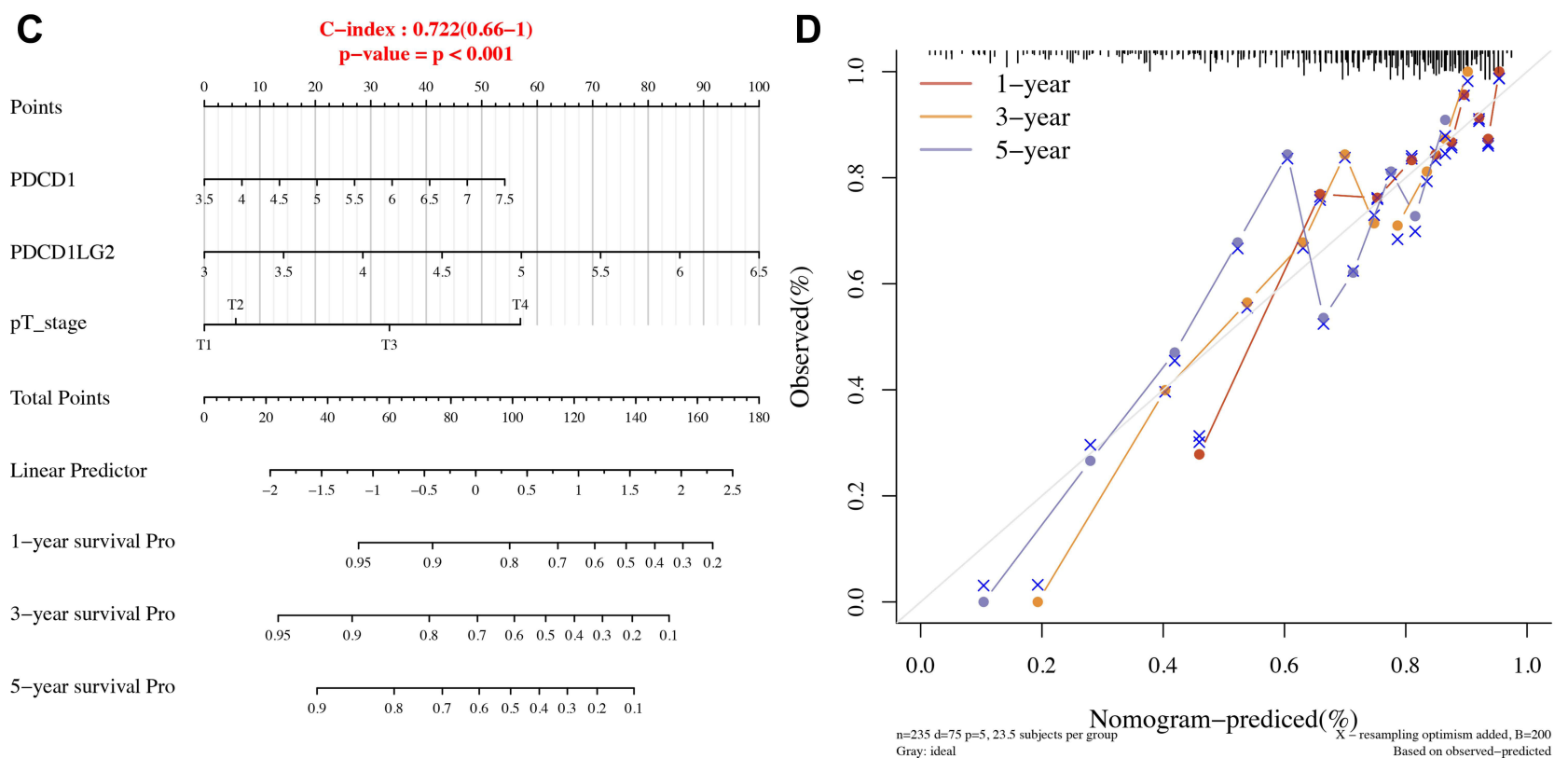

Figure 4 Univariate and multivariate cox regression of immune checkpoints in HCC. (A and B) Univariate and multivariate cox regression of clinical parameters and immune checkpoints in HCC. (C and D) The predictive nomogram to predict the I-y, 3-y and 5-y overall survival of HCC patients.
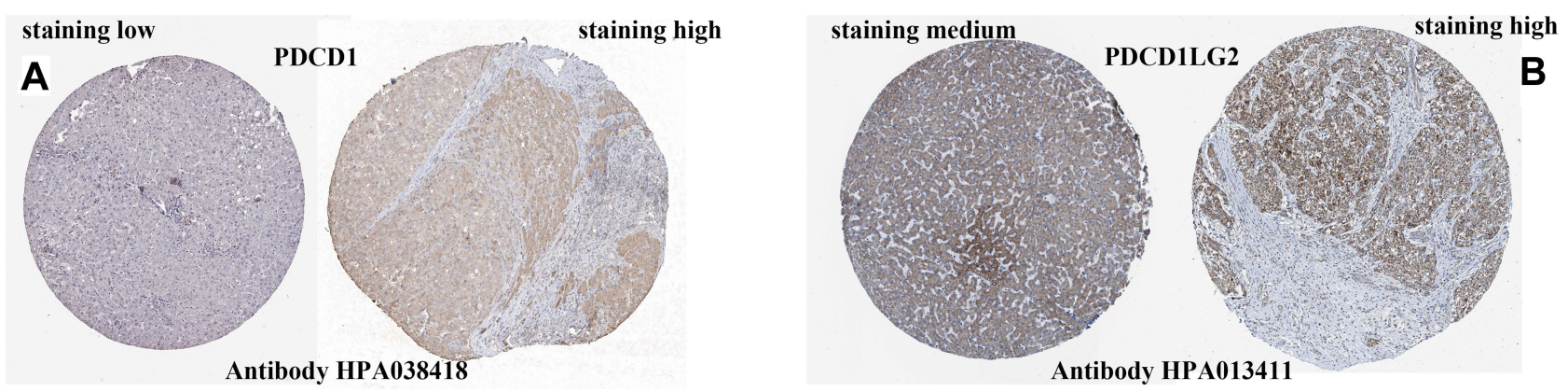

Figure 5 The protein expression of PDCDI/PDCDILG2 in HCC and normal liver tissues. (A) The protein expression of PDCDI in HCC and normal liver tissues. (B) The protein expression of PDCDILG2 in HCC and normal liver tissues. 
A

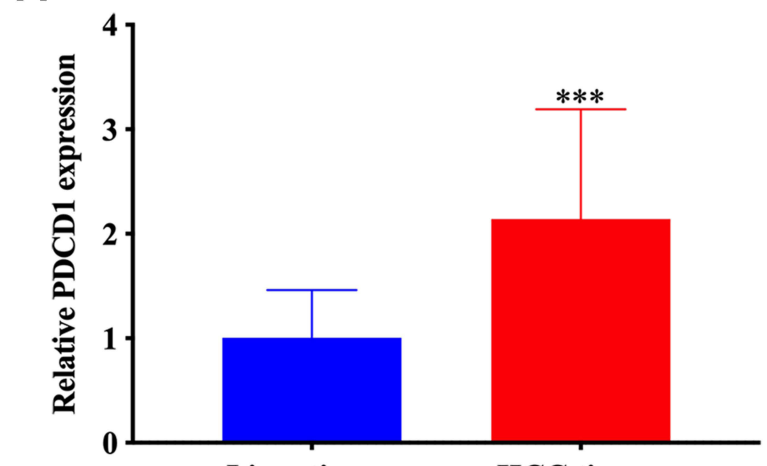

C

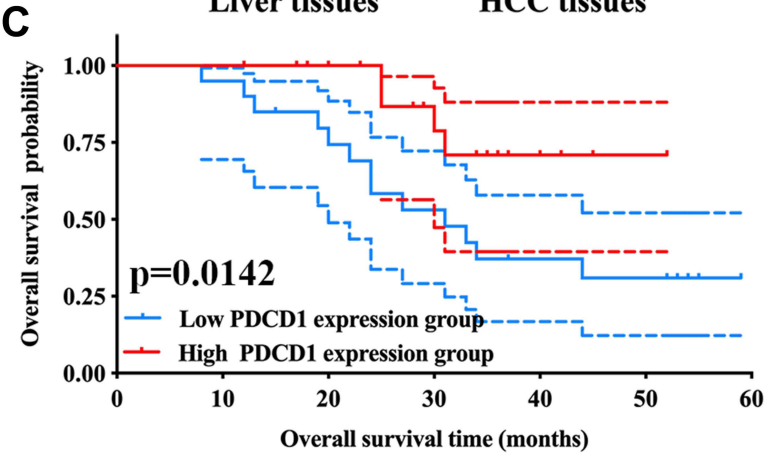

E

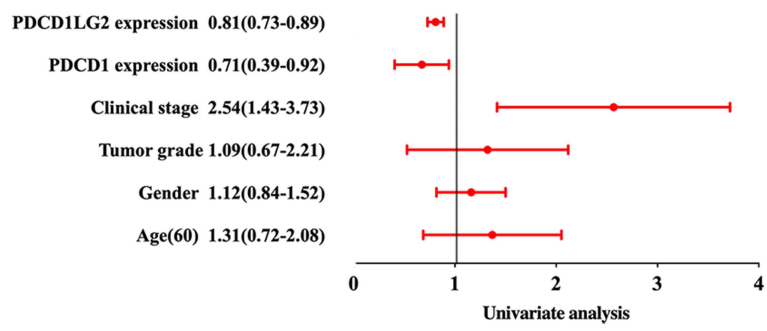

B

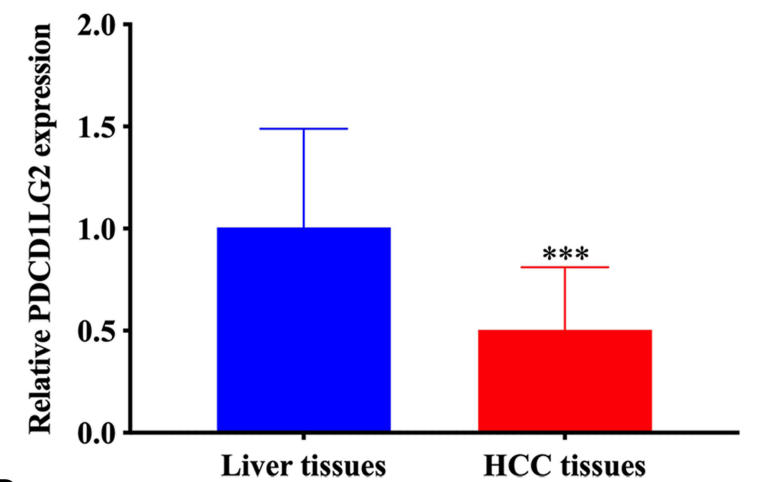

D

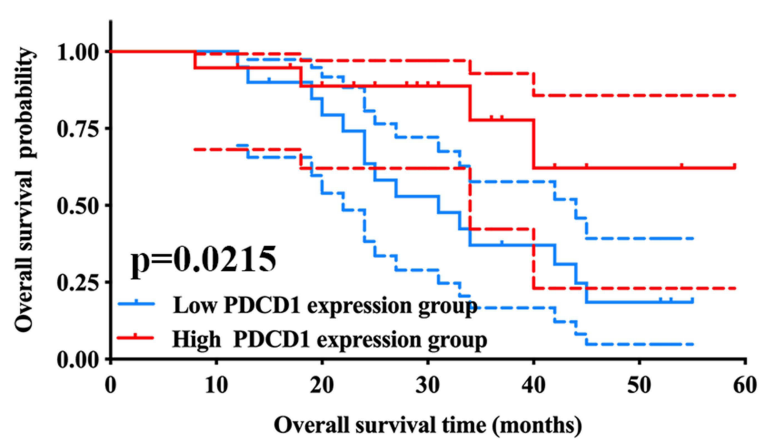

$\mathbf{F}$

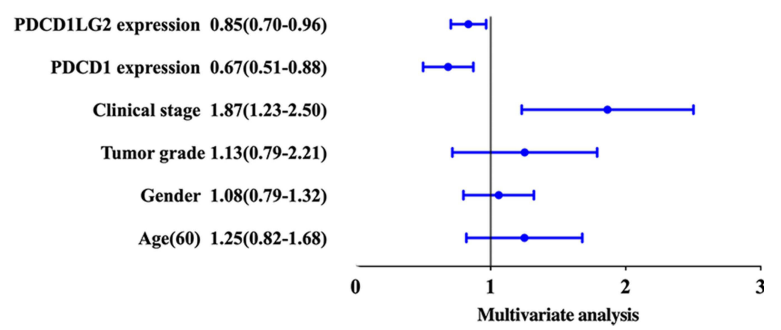

Figure 6 Validation of the expression and overall survival of PDCDI and PDCDILG2 in HCC. (A and B) The mRNA level of PDCDI was upregulated while the mRNA level of PDCDILG2 was downregulated in HCC versus liver tissues. (C and D) HCC patients with high PDCDI and PDCDILG2 expression had a better overall survival. (E and F) Univariate and multivariate analysis demonstrated PDCDI, PDCDILG2 and clinical stage as prognosis factor affecting the OS of HCC patients. ***P < $0.00 \mathrm{I}$.

\section{The Relationship Between PDCDI/ PDCDILG2 and Immune Infiltration in Human Colorectal Cancer (HCC)}

With respect to cancer patients, immune infiltration might serve as a indicator to predict the condition of sentinel lymph nodes and the likelihood of survival. ${ }^{10-12}$ Hence, we probed into the relationship between PDCD1/PDCD1LG2 expression and immune cell infiltration in HCC, which indicated a positive relationship between $\mathrm{PDCD} 1$ and the abundance of CD8 $+\mathrm{T}$ cells $(\mathrm{p}=1.64 \mathrm{e}-32$, Cor $=0.583), \mathrm{B}$ cells $(\mathrm{p}=2 \mathrm{e}-30$, Cor $=0.565), \quad$ CD4 $+\mathrm{T}$ cells $(\mathrm{p}=4.51 \mathrm{e}-12, \quad$ Cor $=0.362)$, Neutrophils $\quad(\mathrm{p}=6.91 \mathrm{e}-12, \quad$ Cor $=0.358), \quad$ Macrophage $(\mathrm{p}=1.32 \mathrm{e}-15$, Cor $=0.415)$ and Dendritic cells $(\mathrm{p}=1.04 \mathrm{e}-30$, Cor $=0.57$ ) (Figure 7A). As illustrated in Figure 7B,
PDCD1LG2 had a positive association with abundance of B cells $(\mathrm{p}=1.31 \mathrm{e}-18$, Cor $=0.451), \mathrm{CD} 4+\mathrm{T}$ cells $(\mathrm{p}=5.26 \mathrm{e}-06$, Cor $=0.243), \quad$ CD8 $+\mathrm{T}$ cells $(\mathrm{p}=8.07 \mathrm{e}-31, \quad$ Cor $=0.57)$, Macrophage $(\mathrm{p}=2.57 \mathrm{e}-16$, Cor $=0.424)$, Dendritic cells $(p=1.59 \mathrm{e}-37, \quad$ Cor $=0.62)$ and Neutrophils $(p=2.43 \mathrm{e}-24$, Cor=0.511). Somatic cell copy number alteration of PDCD1/PDCD1LG2 had little impact on the level of immune cell infiltration (Figure 7C and D). Moreover, further investigation demonstrated a favorable association between the expression of PDCD1/PDCD1LG2 and the expression of immune indicators, such as the indicators of $\mathrm{T}$ cells (general), B cells, follicular helper $\mathrm{T}$ (Tfh) cells, TAMs, monocytes, dendritic cells (DCs), neutrophils, M2 macrophages, T-helper 1 (Th1) cells, T-helper 2 (Th2) cells, M1 macrophages, T-helper 17 (Th17) cells, natural killer 


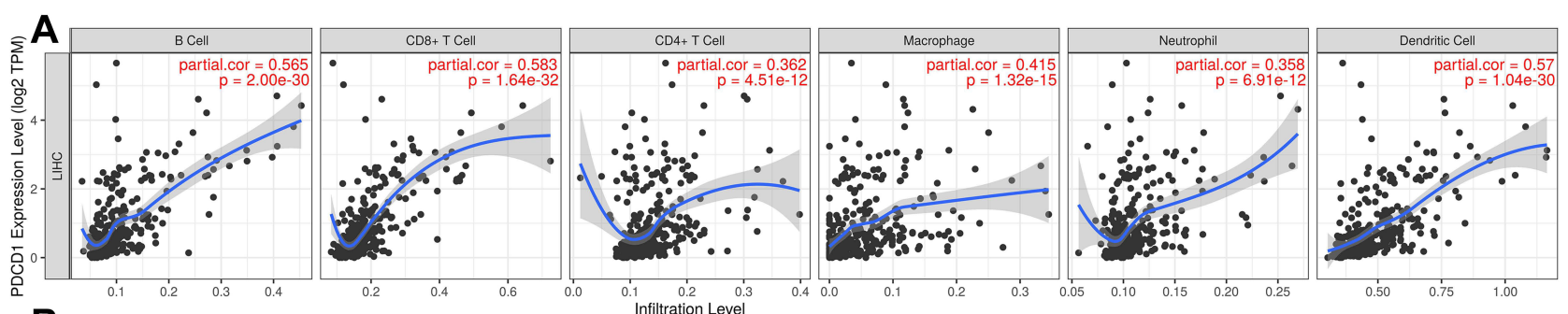

B
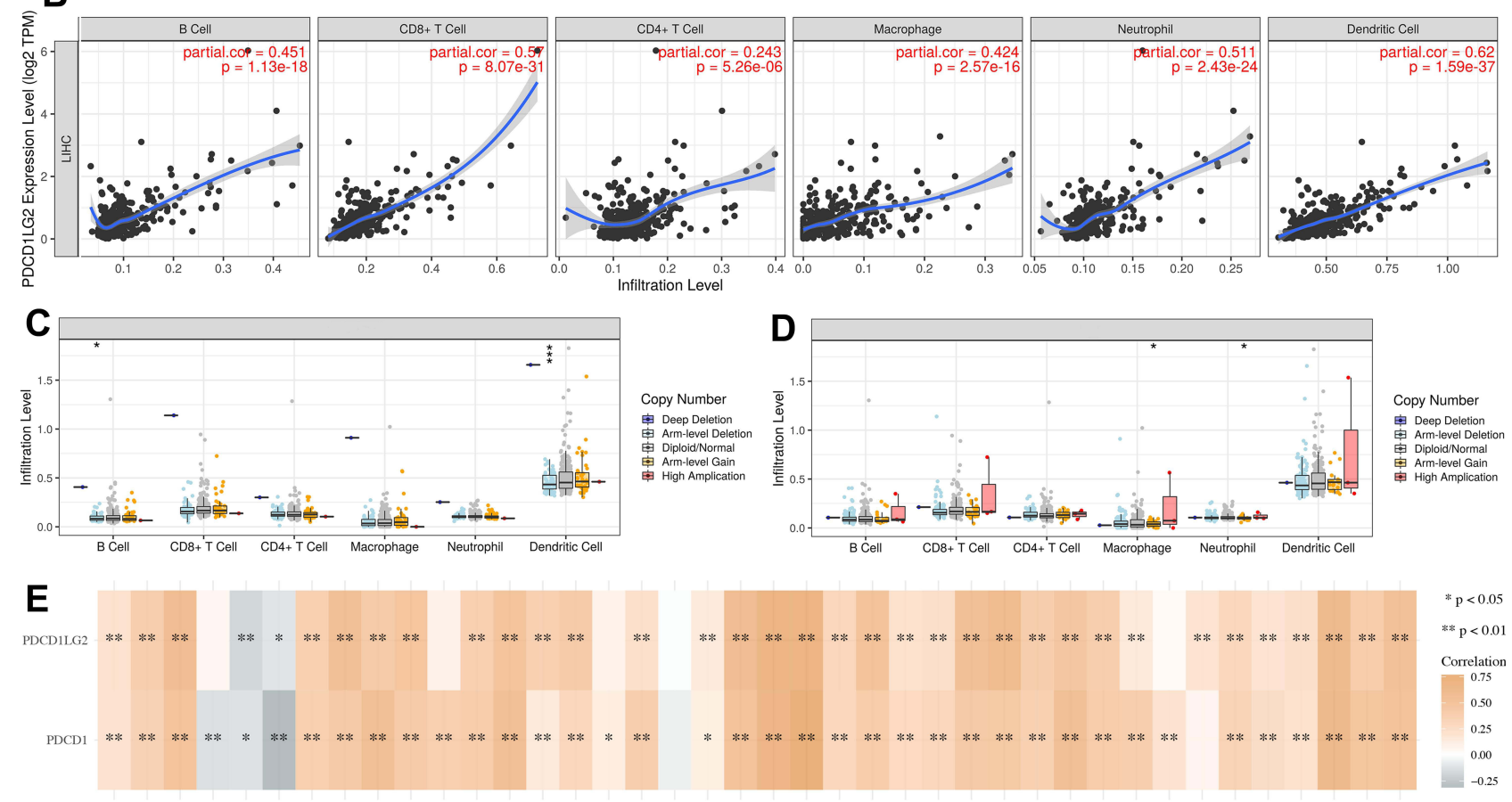

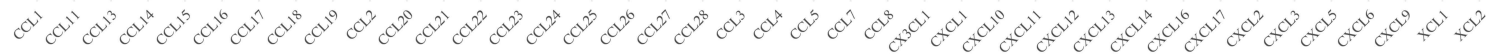

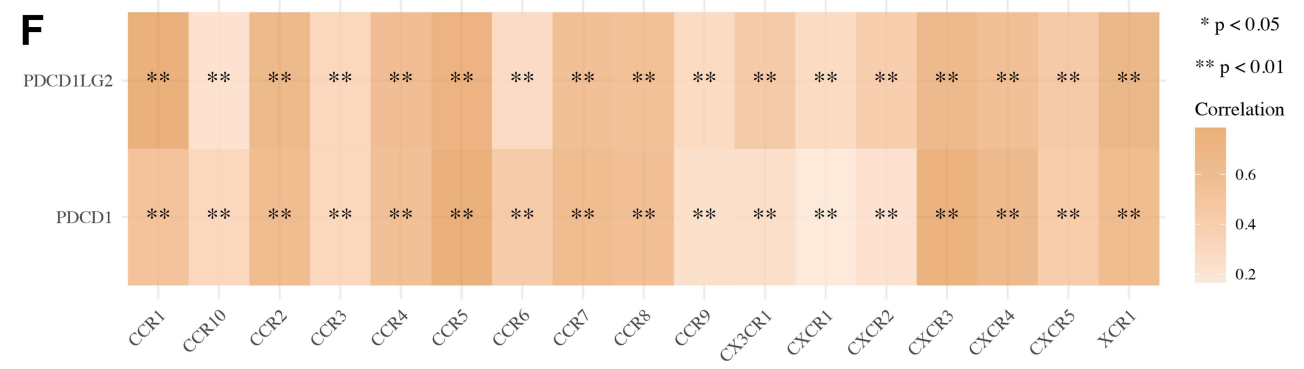

Figure 7 The correlation between immune checkpoints and immune infiltration in HCC. (A and $\mathbf{B})$ The correlation between the expression of PDCDI/PDCDILG2 and the abundance of CD8+ T cells, CD4+ T cells, Macrophage, Neutrophils and Dendritic cells. (C and D) The correlation between SCNA of PDCDI/PDCDILG2 and immune cell infiltration. (E and F) The correlation between the expression of PDCDI/PDCDILG2 and chemokine and chemokine receptor expression. $* \mathrm{P}<0.05$, $* * \mathrm{P}<0.0 \mathrm{I}$, $* * * \mathrm{P}<0.00 \mathrm{I}$. Abbreviation: SCNA, somatic copy number alterations.

(NK) cells, CD8 $+\mathrm{T}$ cells, Tregs, and exhausted $\mathrm{T}$ cells (Table 3). Moreover, we also analyzed the correlation between chemokines and PDCD1/PDCD1L as well as chemokines receptors, indicating that PDCD1/PDCD1LG2 had a positive association with the content of chemokines receptors and chemokines (Figure 7E and F). These findings suggested a potential association between PDCD1/ PDCD1LG2 and immune infiltrates in HCC.

\section{PDCDI/PDCDILG2 Associated}

\section{Functions}

In order to explore PDCD1/PDCD1LG2 associated functions, we then detected the genes correlated with PDCD1/ PDCD1LG2 in HCC and the result was shown in Supplementary Figure 2A and B. GSEA was performed based on PDCD1/PDCD1LG2 and correlated genes. As a result, we found that PDCD1 in GO analysis was 
Table 3 Correlation Analysis Between PDCDI/PDCDILG2 and Gene Biomarkers of Immune Cells in LIHC

\begin{tabular}{|c|c|c|c|c|c|}
\hline \multirow[t]{3}{*}{ Description } & \multirow[t]{3}{*}{ Gene Markers } & \multicolumn{4}{|c|}{ LIHC } \\
\hline & & \multicolumn{2}{|c|}{ PDCDI } & \multicolumn{2}{|c|}{ PDCDILG2 } \\
\hline & & Cor & P-value & Cor & P-value \\
\hline \multirow[t]{2}{*}{ CD8+ T cell } & CD8A & 0.808 & $* * *$ & 0.666 & $* * *$ \\
\hline & CD8B & 0.763 & $* * *$ & 0.601 & $* * *$ \\
\hline \multirow[t]{3}{*}{ T cell (general) } & CD3D & 0.801 & $* * *$ & 0.567 & $* * *$ \\
\hline & CD3E & 0.8 & $* * *$ & 0.678 & $* * *$ \\
\hline & $\mathrm{CD} 2$ & 0.806 & $* * *$ & 0.655 & $* * *$ \\
\hline \multirow[t]{2}{*}{ B cell } & CDI9 & 0.57 & $* * *$ & $0.45 I$ & $* * *$ \\
\hline & CD79A & 0.708 & $* * *$ & 0.546 & $* * *$ \\
\hline \multirow[t]{2}{*}{ Monocyte } & CD86 & 0.675 & $* * *$ & 0.754 & $* * *$ \\
\hline & CDII5(CSFIR) & 0.58 & $* * *$ & 0.743 & $* * *$ \\
\hline \multirow[t]{3}{*}{ TAM } & $\mathrm{CCL} 2$ & $0.4 I I$ & $* * *$ & 0.496 & $* * *$ \\
\hline & CD68 & 0.495 & $* * *$ & $0.55 I$ & $* * *$ \\
\hline & ILIO & 0.534 & $* * *$ & 0.61 & $* * *$ \\
\hline \multirow[t]{3}{*}{ MI Macrophage } & INOS (NOS2) & -0.024 & 0.641 & 0.135 & $* *$ \\
\hline & IRF5 & 0.235 & $* * *$ & 0.199 & $* * *$ \\
\hline & COX2(PTGS2) & 0.353 & $* * *$ & 0.465 & $* * *$ \\
\hline \multirow[t]{3}{*}{ M2 Macrophage } & CDI63 & 0.431 & $* * *$ & 0.762 & $* * *$ \\
\hline & VSIG4 & 0.401 & $* * *$ & 0.652 & $* * *$ \\
\hline & MS4A4A & 0.487 & $* * *$ & 0.76 & $* * *$ \\
\hline \multirow[t]{3}{*}{ Neutrophils } & CD66b (CEACAM8) & 0.085 & 0.103 & 0.091 & 0.0813 \\
\hline & CDIIb (ITGAM) & 0.377 & $* * *$ & 0.502 & $* * *$ \\
\hline & CCR7 & 0.605 & $* * *$ & 0.569 & $* * *$ \\
\hline \multirow[t]{7}{*}{ Natural killer cell } & KIR2DLI & 0.076 & 0.143 & 0.188 & $* * *$ \\
\hline & KIR2DL3 & 0.306 & $* * *$ & 0.321 & $* * *$ \\
\hline & KIR2DL4 & 0.47 & $* * *$ & 0.452 & $* * *$ \\
\hline & KIR3DLI & 0.142 & $* *$ & 0.335 & $* * *$ \\
\hline & KIR3DL2 & 0.395 & $* * *$ & 0.353 & $* * *$ \\
\hline & KIR3DL3 & 0.214 & $* * *$ & 0.189 & $* * *$ \\
\hline & KIR2DS4 & 0.199 & $* * *$ & 0.247 & $* * *$ \\
\hline \multirow[t]{7}{*}{ Dendritic cell } & HLA-DPBI & 0.649 & $* * *$ & 0.714 & $* * *$ \\
\hline & HLA-DQBI & 0.61 & $* * *$ & 0.636 & $* * *$ \\
\hline & HLA-DRA & 0.594 & $* * *$ & 0.726 & $* * *$ \\
\hline & HLA-DPAI & 0.604 & $* * *$ & 0.743 & $* * *$ \\
\hline & BDCA-I (CDIC) & 0.456 & $* * *$ & $0.45 \mathrm{I}$ & $* * *$ \\
\hline & BDCA-4(NRPI) & 0.235 & $* * *$ & 0.337 & $* * *$ \\
\hline & CDIIc (ITGAX) & 0.582 & $* * *$ & 0.598 & $* * *$ \\
\hline \multirow[t]{5}{*}{ ThI } & T-bet (TBX2I) & 0.648 & $* * *$ & 0.676 & $* * *$ \\
\hline & STAT4 & 0.476 & $* * *$ & 0.414 & $* * *$ \\
\hline & STATI & 0.483 & $* * *$ & 0.527 & $* * *$ \\
\hline & IFN-g (IFNG) & 0.642 & $* * *$ & 0.537 & $* * *$ \\
\hline & TNF-A (TNF) & 0.569 & $* * *$ & 0.513 & $* * *$ \\
\hline \multirow[t]{4}{*}{ Th2 } & GATA3 & 0.647 & $* * *$ & 0.634 & $* * *$ \\
\hline & STAT6 & 0.068 & 0.194 & 0.169 & $* *$ \\
\hline & STAT5A & 0.484 & $* * *$ & $0.57 \mid$ & $* * *$ \\
\hline & ILI3 & 0.144 & $* *$ & 0.095 & 0.0683 \\
\hline \multirow[t]{2}{*}{ Tfh } & BCL6 & 0.097 & 0.0632 & 0.128 & $*$ \\
\hline & IL2I & 0.259 & $* * *$ & 0.252 & $* * *$ \\
\hline \multirow[t]{2}{*}{ Thl7 } & STAT3 & 0.167 & $* *$ & 0.351 & $* * *$ \\
\hline & ILI7A & 0.1 & 0.054 & 0.048 & 0.354 \\
\hline
\end{tabular}

(Continued) 
Table 3 (Continued).

\begin{tabular}{|c|c|c|c|c|c|}
\hline \multirow[t]{3}{*}{ Description } & \multirow[t]{3}{*}{ Gene Markers } & \multicolumn{4}{|c|}{ LIHC } \\
\hline & & \multicolumn{2}{|c|}{ PDCDI } & \multicolumn{2}{|c|}{ PDCDILG2 } \\
\hline & & Cor & P-value & Cor & P-value \\
\hline \multirow[t]{4}{*}{ Treg } & FOXP3 & 0.294 & $* * *$ & 0.499 & $* * *$ \\
\hline & CCR8 & 0.546 & $* * *$ & 0.534 & $* * *$ \\
\hline & STAT5B & -0.016 & 0.756 & 0.153 & $* *$ \\
\hline & TGFb (TGFBI) & 0.55 & $* * *$ & 0.404 & $* * *$ \\
\hline \multirow[t]{5}{*}{$\mathrm{T}$ cell exhaustion } & PD-I (PDCDI) & $\mathrm{I}$ & $* * *$ & 0.539 & $* * *$ \\
\hline & CTLA4 & 0.8 & $* * *$ & 0.556 & $* * *$ \\
\hline & LAG3 & 0.675 & $* * *$ & 0.494 & $* * *$ \\
\hline & TIM-3 (HAVCR2) & 0.654 & $* * *$ & 0.699 & $* * *$ \\
\hline & GZMB & 0.583 & $* * *$ & 0.564 & $* * *$ \\
\hline
\end{tabular}

Notes: ${ }^{p}<0.05, * *_{p}<0.01, * * * p<0.001$

involved in $\mathrm{T}$ cell activation, adaptive immune response, leukocyte proliferation, B cell activation, transcription factor activity, monooxygenase activity, cytokine receptor activity, and cytokine binding (Figure 8A-C). PDCD1 in KEGG analysis was involved in Th17 cell differentiation, $\mathrm{T}$ cell receptor signaling pathway, Hematopoietic cell lineage, Drug metabolism, Chemical carcinogenesis, and Th1 and Th2 cell differentiation, (Figure 8D). Moreover, we found that PDCD1LG2 in GO analysis was involved in cellular defense response, cytokine metabolic process, interferon-gamma production, leukocyte proliferation, adaptive immune response, coreceptor activity, cytokine binding, antigen binding, and cytokine receptor activity (Supplementary Figure 3A-C). PDCD1LG2 in KEGG analysis was implicated in TNF signaling pathway, Th1 and Th2 cell differentiation, NOD-like receptor signaling
A

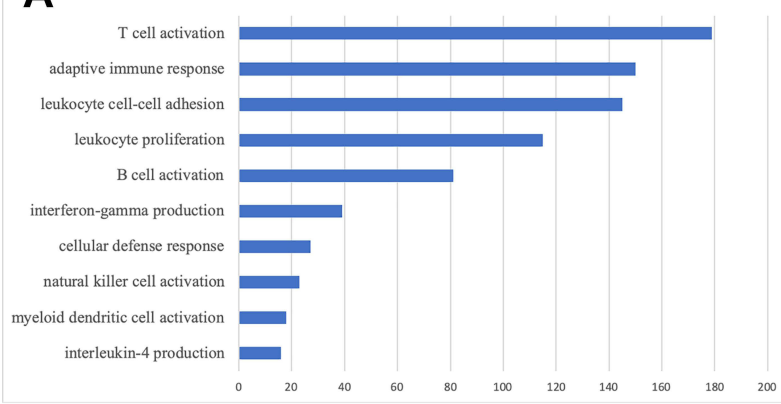

C

MF

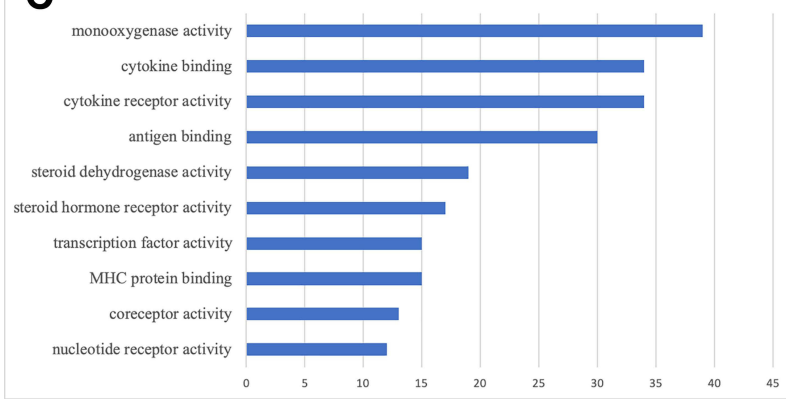

B

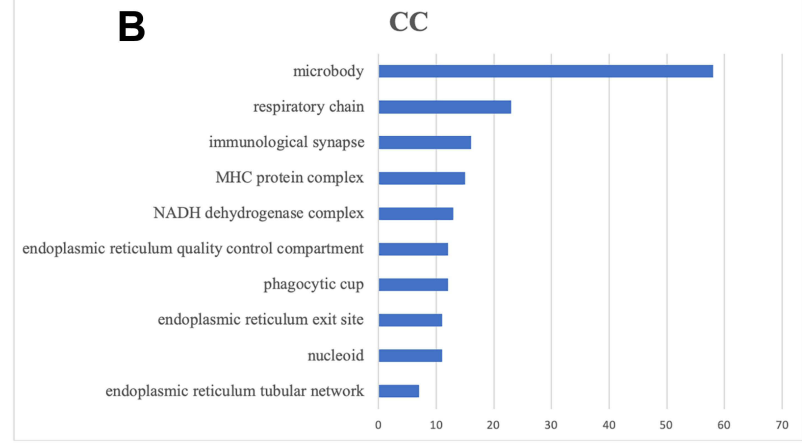

$\mathbf{D}$

\section{KEGG}

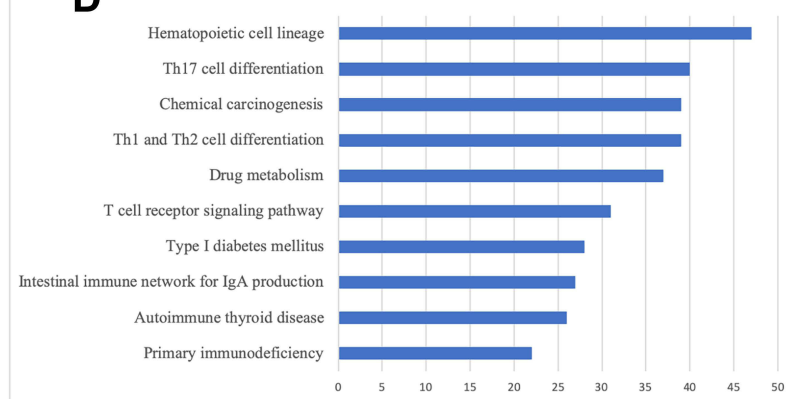

Figure 8 Functional enrichment analysis of PDCDI HCC. (A) The enriched item in biological processes analysis. (B) The enriched item in cellular components analysis. (C) The enriched item in molecular functions analysis. (D) The enriched item in Kyoto Encyclopedia of Genes and Genomes analysis. 
pathway, cell adhesion molecules (CAMs), Th17 cell differentiation, as well as chemokine signaling pathway (Supplementary Figure 3D).

\section{Discussion}

Immune checkpoints are essential in a variety of biological processes and have been shown to improve cancer patients' clinical outcomes. ${ }^{13,14}$ In addition, they have also been reported in a growing body of research to be prognostic indicators in a variety of malignancies, including renal cell carcinoma and lung carcinoma. ${ }^{15-17}$ Whether immune checkpoints might function as a prognostic biomarker in HCC and their relationship to immune infiltration were still unknown. Hence, the objective of the present research was to determine the significance of these immune checkpoints in both immune infiltration and prognosis of HCC patients.

First, we explored the mRNA expression of these immune checkpoints in $\mathrm{HCC}$, indicating that the mRNA levels of SIGLEC15, PDCD1LG2, LAG3, PDCD1, PDCD1LG2, and CTLA4 were increased in HCC tissues as opposed to liver tissues. Notably, the majority of these immune checkpoints were changed in HCC, suggesting that they may perform a critical function in the occurrence and progression of HCC. Furthermore, additional investigations demonstrated that immune checkpoints in HCC were related to the activation of the apoptotic signaling pathway. Apoptosis is an orderly and coordinated cellular process that occurs under physiological and pathological conditions. ${ }^{18}$ Activation of apoptosis could inhibit the carcinogenesis and progression of cancer.

Prognosis analysis revealed that PDCD1 and PDCD1LG2 were prognostic biomarkers for HCC and high PDCD1 and PDCD1LG2 expression had a better RFS, OS, DSS, and PFS. A growing body of research evidence has demonstrated that some immune checkpoints may serve as markers for predicting the prognosis of cancers patients. PDCD1 was a prognosis biomarker in multiple cancers and the high PDCD1 expression was related to an unfavorable OS, uveal melanoma, and DSS in brain glioma. ${ }^{19}$ PDCD1 was shown to be an immunotherapy target and a prognostic marker in non-small cell lung cancer. $^{20}$ HAVCR2 was reported to be a predictive biomarker for gastric cancer and was discovered to be adversely correlated with $\mathrm{OS}^{21}$

Our study also found that PDCD1/PDCD1LG2 showed a favorable relationship with $\mathrm{CD} 8+\mathrm{T}$ cells, $\mathrm{B}$ cells, Macrophage, CD4+ $\mathrm{T}$ cells, Dendritic cells, and
Neutrophils. These immune cells were a vital part of tumor microenvironments and exert a central role in tumor immune infiltration as well as biological mechanisms. Neutrophils could predict poor prognosis in HCC and promote tumor progression through p53 and STAT3 signaling pathways. $^{22}$ Moreover, tumor-associated Neutrophils recruit Macrophages that might facilitate the HCC progression and the formation of sorafenib resistance. $^{23}$ Positive associations were discovered between the expression of PDCD1/PDCD1LG2 and the expression of immune biomarkers, chemokines, and chemokine receptors. Actually, these immune biomarkers, chemokine, and chemokine receptors could serve as a prognostic biomarker and performs an essential function in tumor immune infiltration. CCL2 is a prognostic biomarker and blockade of CCL2/CCR2 signaling in HCC, could inhibit tumor growth by activating $\mathrm{T}$ cell anti-tumor immune response. ${ }^{24}$ Furthermore, in the case of HCC, CCL14 is a predictive biomarker that has been shown to be associated with immune infiltrates. ${ }^{25}$

Our study also has some limitations. First of all, most of the analysis of our study was performed on mRNA level, without protein level. Moreover, animal and in vitro studies should be performed to validate the function of PDCD1/PDCD1LG2 in the occurrence and progression of HCC, as well as its correlation with the infiltration of the immune cells into the microenvironment of the tumor.

\section{Conclusion}

To conclude, the present research performed a thorough investigation of the prognostic significance of immune checkpoints in $\mathrm{HCC}$ as well as their correlation with immune infiltration, which suggested that PDCD1 and PDCD1LG2 were prognostic biomarkers in HCC and related to immune infiltration.

\section{Abbreviations}

HCC, hepatocellular carcinoma; GO, Gene Ontology; KEGG, Kyoto Encyclopedia of Genes and Genomes; BP, biological processes; $\mathrm{CC}$, cellular components; MF, molecular functions; GSEA, Gene Set Enrichment Analysis; OS, overall survival.

\section{Data Sharing Statement}

The analyzed data sets generated during the study are available from the corresponding author on reasonable request. 


\section{Ethics Approval and Consent to Participate}

Our study was approved by the Ethics Committee of Tianjin Medical University Cancer Institute and Hospital. Informed consent was signed by all participating patients.

\section{Disclosure}

The authors declare that they have no competing interests.

\section{References}

1. Ally A, Balasundaram M, Carlsen R. Comprehensive and integrative genomic characterization of hepatocellular carcinoma. Cell. 2017;169 (7):1327-1341.e23. doi:10.1016/j.cell.2017.05.046

2. Vogel A, Cervantes A, Chau I, et al. Hepatocellular carcinoma: ESMO clinical practice guidelines for diagnosis, treatment and follow-up. Ann Oncol. 2019;30(5):871-873. doi:10.1093/annonc/ mdy 510

3. Yang JD, Hainaut P, Gores GJ, et al. A global view of hepatocellular carcinoma: trends, risk, prevention and management. Nat Rev Gastroenterol Hepatol. 2019;16(10):589-604. doi:10.1038/s41575019-0186-y

4. Zhang Y, Zheng J. Functions of immune checkpoint molecules beyond immune evasion. Adv Exp Med Biol. 2020;1248:201-226.

5. Lo Re O, Mazza T, Giallongo S, et al. Loss of histone macroH2A1 in hepatocellular carcinoma cells promotes paracrine-mediated chemoresistance and $\mathrm{CD} 4(+) \mathrm{CD} 25(+) \mathrm{FoxP} 3(+)$ regulatory $\mathrm{T}$ cells activation. Theranostics. 2020;10(2):910-924. doi:10.7150/th no. 35045

6. Xu F, Jin T, Zhu Y, et al. Immune checkpoint therapy in liver cancer. $J$ Exp Clin Cancer Res. 2018;37(1):110. doi:10.1186/s13046-018$0777-4$

7. Ye Y, Xiang Y, Ozguc FM, et al. The genomic landscape and pharmacogenomic interactions of clock genes in cancer chronotherapy. Cell Syst. 2018;6(3):314-328.e2. doi:10.1016/j. cels.2018.01.013

8. Uhlen M, Zhang C, Lee S, et al. A pathology atlas of the human cancer transcriptome. Science. 2017;357(6352):eaan2507. doi:10.11 26/science.aan 2507

9. Vasaikar SV, Straub P, Wang J, et al. LinkedOmics: analyzing multi-omics data within and across 32 cancer types. Nucleic Acids Res. 2018;46(D1):D956-D963. doi:10.1093/nar/gkx1090

10. Liang F, Liang H, Li Z, et al. JAK3 is a potential biomarker and associated with immune infiltration in kidney renal clear cell carcinoma. Int Immunopharmacol. 2020;86:106706. doi:10.1016/j. intimp.2020.106706

11. Zeng Q, Sun S, Li Y, et al. Identification of therapeutic targets and prognostic biomarkers among CXC chemokines in the renal cell carcinoma microenvironment. Front Oncol. 2020;9:1555. doi: $10.3389 /$ fonc. 2019.01555

International Journal of General Medicine

\section{Publish your work in this journal}

The International Journal of General Medicine is an international, peer-reviewed open-access journal that focuses on general and internal medicine, pathogenesis, epidemiology, diagnosis, monitoring and treatment protocols. The journal is characterized by the rapid reporting of reviews, original research and clinical studies
12. Zhou L, Li Y, Li Z, et al. Mining therapeutic and prognostic significance of STATs in renal cell carcinoma with bioinformatics analysis. Genomics. 2020;112(6):4100-4114. doi:10.1016/j.yge no.2020.06.032

13. Adda L, Batteux B, Saidak Z, et al. Effect of immune checkpoint inhibitor-induced rheumatic and musculoskeletal disorders on overall survival. Joint Bone Spine. 2021:105168. doi:10.1016/j.jbspin.2 021.105168

14. Maj T, Wang W, Crespo J, et al. Oxidative stress controls regulatory $\mathrm{T}$ cell apoptosis and suppressor activity and PD-L1-blockade resistance in tumor. Nat Immunol. 2017;18(12):1332-1341. doi:10.1038/ ni. 3868

15. Paderi A, Giorgione R, Giommoni E, et al. Association between immune related adverse events and outcome in patients with metastatic renal cell carcinoma treated with immune checkpoint inhibitors. Cancers. 2021;13(4):860. doi:10.3390/cancers 13040860

16. Grandal B, Mangiardi-Veltin M, Laas E, et al. PD-L1 expression after neoadjuvant chemotherapy in triple-negative breast cancers is associated with aggressive residual disease, suggesting a potential for immunotherapy. Cancers. 2021;13(4):746. doi:10.3390/cancers1 3040746

17. Darvin P, Toor SM, Sasidharan Nair V, et al. Immune checkpoint inhibitors: recent progress and potential biomarkers. Exp Mol Med. 2018;50(12):1-11. doi:10.1038/s12276-018-0191-1

18. Wong RS. Apoptosis in cancer: from pathogenesis to treatment. $J$ Exp Clin Cancer Res. 2011;30(1):87. doi:10.1186/1756-9966-30-87

19. Miao Y, Wang J, Li Q, et al. Prognostic value and immunological role of PDCD1 gene in pan-cancer. Int Immunopharmacol. 2020;89(Pt B):107080. doi:10.1016/j.intimp.2020.107080

20. Tsoukalas N, Kiakou M, Tsapakidis K, et al. PD-1 and PD-L1 as immunotherapy targets and biomarkers in non-small cell lung cancer. J BUON. 2019;24(3):883-888.

21. Wang Y, Zhao E, Zhang Z, et al. Association between Tim-3 and Gal-9 expression and gastric cancer prognosis. Oncol Rep. 2018;40 (4):2115-2126. doi:10.3892/or.2018.6627

22. Wang Y, Yao R, Zhang D, et al. Circulating neutrophils predict poor survival for HCC and promote HCC progression through p53 and STAT3 signaling pathway. $J$ Cancer. 2020;11(13):3736-3744. doi: $10.7150 /$ jca. 42953

23. Zhou S-L, Zhou Z-J, Hu Z-Q, et al. Tumor-associated neutrophils recruit macrophages and T-regulatory cells to promote progression of hepatocellular carcinoma and resistance to sorafenib. Gastroenterology. 2016;150(7):1646-1658.e17. doi:10.1053/j. gastro.2016.02.040

24. Li X, Yao W, Yuan Y, et al. Targeting of tumour-infiltrating macrophages via CCL2/CCR2 signalling as a therapeutic strategy against hepatocellular carcinoma. Gut. 2017;66(1):157-167. doi:10.1136/ gutjnl-2015-310514

25. Lyu L, Zheng Y, Hong Y, et al. Comprehensive analysis of the prognostic value and immune function of chemokine-CXC receptor family members in breast cancer. Int Immunopharmacol. 2020;87:106797. doi:10.1016/j.intimp.2020.106797 across all disease areas. The manuscript management system is completely online and includes a very quick and fair peer-review system, which is all easy to use. Visit http://www.dovepress.com/ testimonials.php to read real quotes from published authors. 